\title{
Abordaje posterior mínimamente invasivo en artroplastia total de cadera. Estudio prospectivo y aleatorizado. Un año de seguimiento
}

\section{Minimally invasive posterior approach in total hip arthroplasty. Prospective randomised trial}

\author{
J.R. Varela-Egocheaga ${ }^{1}$, M.A. Suárez-Suárez ${ }^{2,4}$, M. Fernández-Villán², V. González-Sastre ${ }^{2}$, \\ J.R. Varela-Gómez ${ }^{3}$, A. Murcia-Mazón ${ }^{2,4}$
}

\section{RESUMEN}

Fundamento. La hipótesis de trabajo fue que la cirugía mínimamente invasiva era superior a la convencional para el proceso de artroplastia total de cadera. Para ello se compararon los resultados de la cirugía mínimamente invasiva con los de la cirugía convencional en artroplastia total de cadera.

Material y métodos. Ensayo clínico prospectivo y aleatorizado. Se seleccionaron 50 pacientes, los cuales fueron divididos en dos grupos en función del abordaje quirúrgico: posterior mínimamente invasivo o posterior directo convencional. Se evaluaron el sangrado perioperatorio, el dolor postoperatorio, el tiempo de recuperación, la orientación y ajuste de los componentes, la tasa de complicaciones y el resultado funcional y se han seguido a los pacientes un año mínimo.

Resultados. No se han encontrado diferencias significativas entre los grupos en cuanto a sangrado perioperatorio. El dolor postoperatorio fue menor con la cirugía mínimamente invasiva, la velocidad de recuperación fue significativamente mayor con el abordaje posterior mínimamente invasivo al detectarse una menor estancia hospitalaria y un inicio más precoz de la deambulación. No se encontraron diferencias en cuanto a tiempo quirúrgico, orientación y ajuste de los componentes, tasa de complicaciones ni resultado funcional. Impacto económico favorable a la cirugía mínimamente invasiva con un ahorro del $5 \%$ del total de coste del proceso.

Conclusiones. El abordaje posterior mínimamente invasivo disminuye el dolor y acelera la recuperación con un impacto económico favorable, sin mostrar diferencias en ninguna de los demás aspectos estudiados.

Palabras clave. Prótesis total de cadera. Cirugía mínimamente invasiva. Abordaje posterior

An. Sist. Sanit. Navar. 2010; 33 (2): 133-143

\begin{abstract}
Backgrond. Our working hypothesis was that minimally invasive surgery was superior to conventional surgery for total hip arthroplasty procedure. We compared THR results in the minimally invasive posterior approach with THR results in the conventional posterior approach.
\end{abstract}

Methods. Prospective, randomised trial. Fifty patients were selected and then divided into two groups based on treatment (minimally invasive posterior approach or conventional posterior approach). Data collected: Perioperative bleeding, postoperative pain, time of recovery, component orientation, complications and functional results. One year follow-up.

Results. No differences were found in blood loss, surgical time, component orientation, rate of complications or functional result. The minimally invasive lateral approach produced less postoperative pain and a faster recovery, with a shorter hospital stay and earlier walking-start, and a positive economic impact with $5 \%$ of the total process cost saved.

Conclusions. Minimally invasive surgery permits less postoperative pain, faster recovery and a positive economic saving, without differences in the rest of the items studied.

Key words. Total hip arthroplasty. Minimally invasive surgery. Posterior approach.
1. Servicio de Cirugía Ortopédica y Traumatología. Hospital de Navarra. Pamplona.

2. Servicio de Cirugía Ortopédica y Traumatología. Hospital de Cabueñes. Gijón. Asturias.

3. Servicio de Cirugía Ortopédica y Traumatología. Hospital Álvarez-Buylla. Mieres. Asturias.

4. Universidad de Oviedo.

Recepción: 9 de septiembre de 2009

Aceptación provisional: 19 de enero de 2010

Aceptación definitiva: 4 de febrero de 2010

\author{
Correspondencia \\ José Ramón Varela Egocheaga \\ Servicio de Cirugía Ortopédica y Traumatología \\ Hospital de Navarra \\ Irunlarrea, 3 \\ 31008 Pamplona \\ E-mail: varelaegocheaga@yahoo.es
}




\section{INTRODUCCIÓN}

Cada década ha tenido su controversia en la artroplastia total de cadera. En la década de los 70 se exploraron las posibles ventajas del par de fricción metal-metal frente al convencional metal-polietileno, la década de los 80 vivió el nacimiento de la fijación biológica; en los 90 se investigaron las ventajas entre la fijación distal o proximal de los vástagos femorales y se describieron nuevos pares de fricción. La década del 2000 probablemente será recordada como la del nacimiento del concepto de cirugía mínimamente invasiva ${ }^{1,2}$.

Se pueden definir de forma general las técnicas de cirugía mínimamente invasivas (CMI o MIS) como aquellas en las que se ha disminuido la incisión cutánea y modificado el acceso quirúrgico en un intento de reducir la lesión tisular asociada a cualquier procedimiento $^{3}$. Los principios de la CMI en artroplastia total de cadera son ${ }^{4,5}$ : disminución de la incisión cutánea, creación de una ventana móvil, reducción de la disección profunda conservando la mayor cantidad de tejido muscular en su lugar y utilización de material específico que respete al máximo los tejidos blandos.

Se han descrito muchas vías de abordaje mínimamente invasivas en artroplastia total de cadera. Clásicamente se dividen en dos grupos: de vía única (posterior, lateral directa, anterolateral y anterior), que suelen ser modificaciones de los abordajes clásicos, y de vía múltiple (anterior con portales accesorios y doble vía).

Como se ha comentado, a medio y largo plazo la artroplastia total de cadera ha demostrado ser eficaz en la disminución del dolor y la mejora de la función, calidad de vida y salud general; sin embargo, a corto plazo la amplia disección necesaria para la colocación de la prótesis se traduce en dolor y disminución de la función que retrasa la completa recuperación. Las técnicas de cirugía mínimamente invasivas pueden tener beneficios potenciales para el paciente, de hecho, en otras áreas de la cirugía ortopédica se ha demostrado científicamente, mediante evaluación de la elevación de los reactantes de fase aguda, que la agresión tisular es significativamente menor con las técnicas de cirugía MIS ${ }^{6}$ y esta disminución de la agresión podría conducir a ventajas en cuanto al sangrado perioperatorio, dolor postoperatorio y velocidad de recuperación ${ }^{7,8}$.

La cirugía mínimamente invasiva en artroplastia total de cadera está de plena actualidad, como lo demuestra el hecho de que en el año 2008 se publicaran nada menos que 59 artículos científicos sobre ella. Sin embargo, apenas existen trabajos con un diseño metodológico que pueda proporcionar un alto nivel de evidencia científica, la mayoría de los artículos y citas que se pueden encontrar tanto en buscadores generales como específicos de cirugía ortopédica son descriptivos, de series de casos, retrospectivos, etc ${ }^{9}$.

La realización de este trabajo pretende contribuir a situar la cirugía mínimamente invasiva para la artroplastia total de cadera en su justo lugar dentro del arsenal de técnicas disponibles para el cirujano ortopédico.

Nuestra pregunta de investigación fue ¿es la cirugía mínimamente invasiva superior a la técnica convencional en la implantación de una artroplastia total de cadera?

El objetivo del presente trabajo ha sido comparar los resultados obtenidos mediante el abordaje posterior mínimamente invasivo y el abordaje posterior convencional en cuanto a sangrado perioperatorio, dolor postoperatorio, velocidad de recuperación, tiempo quirúrgico, orientación y ajuste de los componentes implantados, tasa de complicaciones, resultado funcional e impacto económico.

\section{MATERIAL Y MÉTODOS}

Se diseñó un estudio prospectivo y aleatorizado para el que se seleccionaron, entre junio de 2006 y abril de 2007, 50 pacientes con los siguientes criterios de inclusión: diagnóstico de coxartrosis primaria o secundaria a necrosis aséptica de cabeza femoral, coxartrosis subsidiaria de tratamiento mediante ATC según los crite- 
rios normalmente utilizados, aceptación por parte del paciente de la alternativa terapéutica así como firma del consentimiento informado para la intervención y para su inclusión en el estudio. Se excluyeron del estudio los pacientes con diagnóstico de displasia de desarrollo de la cadera, antecedentes de intervenciones quirúrgicas sobre la cadera a intervenir, intervenidos de ATC contralateral en el año previo a la intervención quirúrgica y/o índice de masa corporal (IMC) mayor de 40. Los pacientes fueron divididos en dos grupos de 25 pacientes cada uno mediante el uso de una tabla de números aleatorizados.

En uno de los grupos (grupo PMIS) se implantó la artroplastia total de cadera mediante un abordaje lateral mínimamente invasivo según técnica de Sculco y col $^{10}$ (Fig. 1).

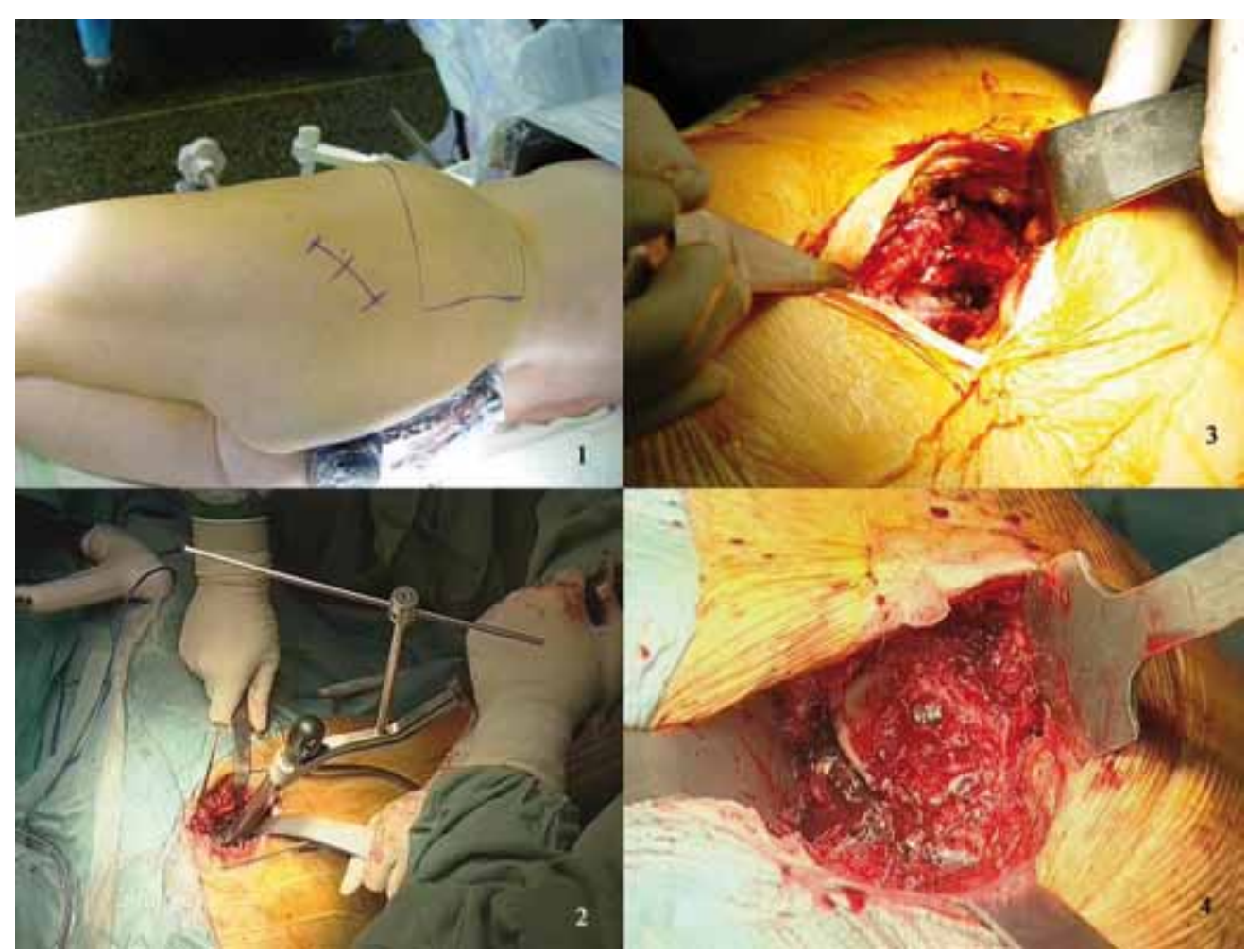

Figura 1. Abordaje posterior mínimamente invasivo.

Se colocó el paciente en decúbito lateral sobre la cadera contralateral manteniendo la pelvis vertical mediante el uso de un posicionador especialmente diseñado a tal efecto. Tras la desinfección y preparación del campo quirúrgico se realizó una incisión cutánea de igual dirección que en el caso del acceso convencional pero menor de 10 centímetros centrada en la punta del trocánter mayor. Se profundizó la incisión hasta el plano aponeurótico incidiéndose éste en la dirección de la herida quirúrgica, se expuso el músculo piriforme y se separó del músculo glúteo menor. Se realizó una desinserción cápsulo-muscular como la anteriormente descrita, pero en este caso sólo de los músculos piriforme y gémino superior, respetando el cuadrado crural y el gémino inferior; en ningún caso se realizó desinserción de la expansión del 
glúteo mayor. El resto de la intervención se realizó de la forma habitual con la excepción de la utilización de material específico para cirugía mínimamente invasiva. En el otro grupo se utilizó el abordaje posterior convencional según técnica de Moore y Gibson. Todos los pacientes fueron intervenidos por el mismo equipo quirúrgico. En todos los pacientes se implantó el mismo modelo protésico: componente acetabular Bihapro® ${ }^{\circledR}$ (Biomet ${ }^{\circledR}$ Bridgend, Reino Unido) con interior de polietileno de ultra-alto peso molecular convencional para cabeza de $28 \mathrm{~mm}$, componente femoral Cerafit ${ }^{\circledR}$ (Ceraver ${ }^{\circledR}$ Gonesse, Francia) no cementado; y cabeza de alúmina de $28 \mathrm{~mm}$ modelo Cerafit ${ }^{\circledR}$ (Ceraver ${ }^{\circledR}$ Gonesse, Francia). En todos los pacientes se utilizaron los mismos separadores y material específico. Todos los pacientes recibieron profilaxis antitrombótica durante 6 semanas y antibiótica durante 24 horas.

El protocolo postoperatorio fue idéntico en ambos grupos; se permitió al paciente sentarse y caminar con la ayuda de dos bastones a partir del día siguiente de la intervención. Se retiraron los drenajes a las 48 horas en todos los pacientes. En cuanto a la medicación analgésica se siguió el siguiente protocolo: se pautó en todos los pacientes metamizol (Nolotil@) a razón de 1 ampolla intravenosa cada 8 horas y 21 miligramos subcutáneos de meperidina (dolantina $\left.{ }^{\circledR}\right)$ de rescate. Esta medicación se administró sólo a demanda del paciente en escala creciente tal como se ha expuesto. Se procedió al alta hospitalaria cuando el paciente se encontraba deambulante y con buen aspecto de la herida quirúrgica. Las variables estudiadas fueron las siguientes:

- Comprobación de homogeneidad de grupos: edad, sexo, lado intervenido, peso, IMC, diagnóstico de indicación, hemoglobina preoperatoria, escala de Harris (Harris Hip Store) preoperatorio y antecedentes personales.

- Sangrado perioperatorio: hemoglobina postoperatoria a las 6 y 48 horas de la intervención, descenso de los valores de hemoglobina des- de el preoperatorio hasta las 6 y 48 horas de la intervención, número de pacientes transfundidos, concentrados de hematíes transfundidos por paciente y drenaje quirúrgico.

- Dolor postoperatorio: número de pacientes que precisaron analgésicos y/o opioides para mitigar el dolor y miligramos de metamizol administrados por paciente en el día 1 y 2 del postoperatorio.

- Velocidad de recuperación: día de inicio de la deambulación (primer día en el que el paciente fue capaz de caminar 10 pasos seguidos) y estancia hospitalaria. Para el estudio del inicio de la deambulación se consideró día 1 como el día siguiente al de la intervención.

- Tiempo quirúrgico: tiempo transcurrido de incisión cutánea a cierre de la piel.

- Orientación de los componentes: ángulo del componente acetabular respecto a la línea bi-isquiática, número de componentes acetabulares colocados horizontales $\left(<35^{\circ}\right)$, neutros $\left(35-45^{\circ}\right)$ o verticales ( $\left.>45^{\circ}\right)$, ángulo del componente femoral respecto al eje diafisario femoral, número de componentes femorales colocados en varo $\left(<177^{\circ}\right)$, neutros $\left(177-183^{\circ}\right)$ o en valgo ( $\left.>183^{\circ}\right)$ (Fig. 2 ).

- Ajuste de los componentes: relleno metafisario y relleno diafisario según método de Barrack y coll1 (Fig. 2) y dismetría clínica mediante la medición desde la espina ilíaca anterosuperior hasta el borde inferior del maleolo interno.

- Tasa de complicaciones: relación de complicaciones acaecidas durante el postoperatorio.

- Resultado funcional: (Harris Hip Store) a los 3 y 12 meses de la intervención quirúrgica.

- Impacto económico: se utilizó el método de minimización de costes. Suponiendo el mismo resultado final en ambos grupos se comparó el gas- 
to producido en cada grupo en tres aspectos principales: estancia hospitalaria, tiempo quirúrgico, concentrados de hematíes transfundidos y complicaciones. Para ello se utilizaron los precios publicados por la gerencia del Hospital de Cabueñes del año 2007.

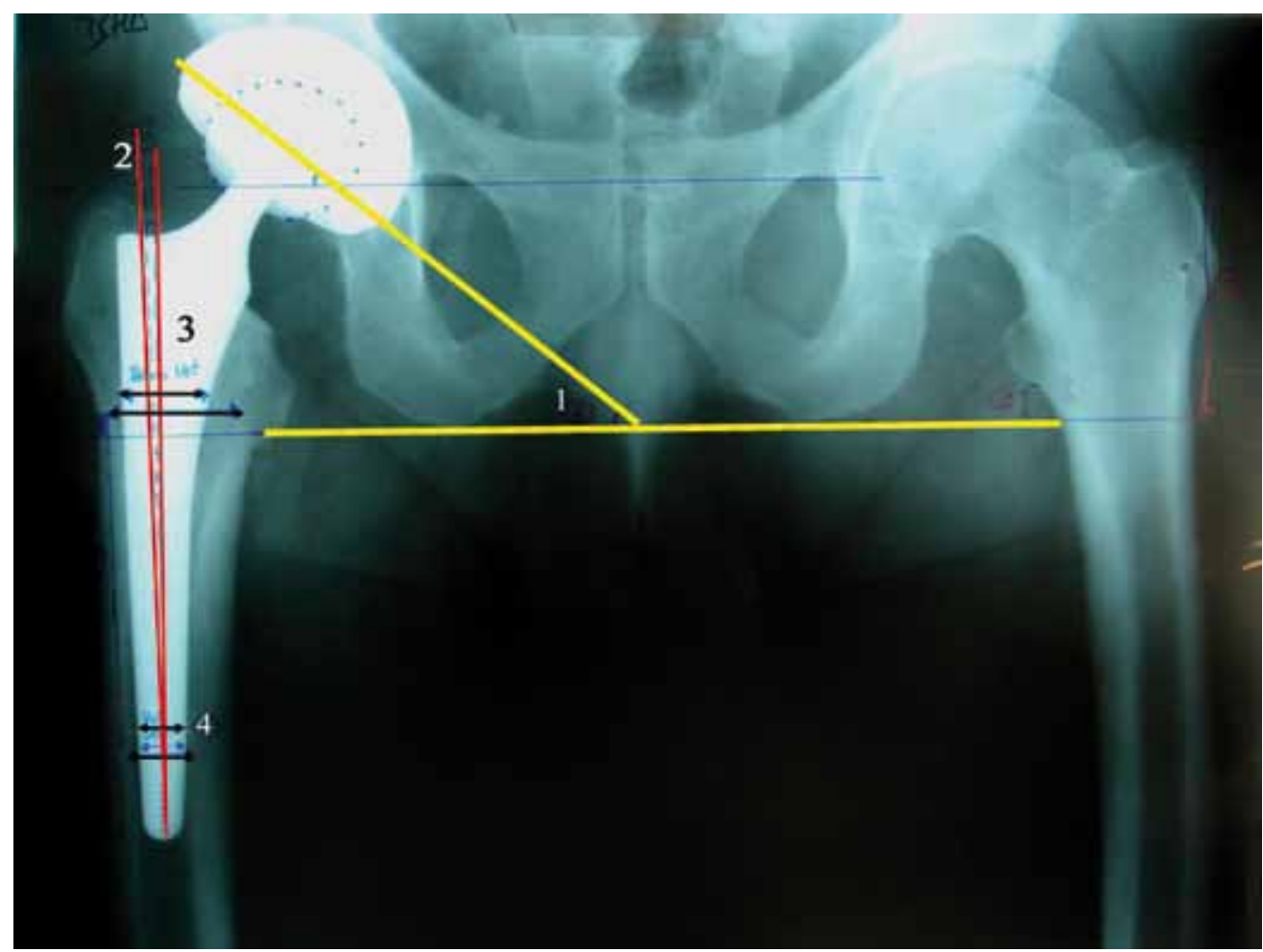

Figura 2. Mediciones radiográficas realizadas (Bihapro-Cerafit).

1. Ángulo del componente acetabular respecto a la línea bi-isquiática.

2. Ángulo del componente femoral respecto al eje diafisario femoral.

3. Relleno metafisario.

4. Relleno diafisario.

En cuanto al análisis estadístico, se aplicó a todos las variables cuantitativas la prueba de Kolmogorov-Smirnov para comprobar su ajuste a la distribución normal y la prueba de Levene de homogeneidad de varianzas. En el caso de ajuste a la distribución normal y homogeneidad de varianzas se aplicó una t de student, mientras que en caso contrario se aplicó una $U$ de Mann-Whitney. Las variables cualitativas se evaluaron mediante la prueba de Chicuadrado. Se consideró la diferencia estadísticamente significativa cuando $\mathrm{p}<0,05$.
El diseño del estudio fue aprobado por el Comité de Ética del Hospital de Cabueñes y todos los pacientes aceptaron, por escrito, ser incluidos en el estudio.

\section{RESULTADOS}

Antes de exponer los resultados del estudio, y tal como se puede observar en la tabla 1, los dos grupos no mostraron diferencias significativas en cuanto a la edad, sexo, lado intervenido, diagnóstico de indicación, peso, IMC, valores preoperatorios 
de hemoglobina ni Harris Hip Score preoperatorio. En cuanto a los antecedentes per- sonales tampoco se detectaron diferencias significativas entre ambos grupos.

Tabla 1. Exposición y comparación de las variables preoperatorias de ambos grupos.

\begin{tabular}{|c|c|c|c|c|c|c|c|c|}
\hline & $\begin{array}{l}\text { Edad } \\
\text { (años) }\end{array}$ & Sexo & Lado & Diagnóstico & $\begin{array}{l}\text { Peso } \\
\text { (Kg) }\end{array}$ & IMC & $\begin{array}{c}\text { Hb Preop } \\
\text { g/dl }\end{array}$ & HHS Preop \\
\hline $\begin{array}{c}\text { Grupo } \\
\text { PMIS }\end{array}$ & $\begin{array}{c}60,76 \\
(11,07)\end{array}$ & $\begin{array}{l}14 \mathrm{H} \\
11 \mathrm{M}\end{array}$ & $\begin{array}{c}17 \mathrm{D} \\
8 \mathrm{I}\end{array}$ & $\begin{array}{c}22 \mathrm{CA} \\
3 \mathrm{NACF}\end{array}$ & $\begin{array}{c}72,16 \\
(11,28)\end{array}$ & $\begin{array}{l}27,04 \\
(3,49)\end{array}$ & $\begin{array}{l}14,28 \\
(1,39)\end{array}$ & $\begin{array}{c}61,04 \\
(17,08)\end{array}$ \\
\hline $\begin{array}{c}\text { Grupo } \\
\text { PEST }\end{array}$ & $\begin{array}{c}61,32 \\
(10,66)\end{array}$ & $\begin{array}{l}11 \mathrm{H} \\
14 \mathrm{M}\end{array}$ & $\begin{array}{c}19 \mathrm{D} \\
6 \mathrm{I}\end{array}$ & $\begin{array}{c}21 \mathrm{CA} \\
4 \mathrm{NACF}\end{array}$ & $\begin{array}{c}79,60 \\
(15,38)\end{array}$ & $\begin{array}{l}29,56 \\
(4,89)\end{array}$ & $\begin{array}{l}13,98 \\
(1,18)\end{array}$ & $\begin{array}{c}55,32 \\
(15,49)\end{array}$ \\
\hline Sig. (p) & $0,983^{\#}$ & 0,852 & 0,324 & 0,222 & $0,076^{\#}$ & $0,093^{\#}$ & $0,230^{\#}$ & $0,708^{\#}$ \\
\hline
\end{tabular}

Entre paréntesis la desviación estándar

PMIS: grupo de abordaje posterior mínimamente invasivo

PEST: grupo de abordaje posterior estándar

H: Hombre; M: Mujer

CA: Coxartrosis; NACF: Necrosis Aséptica Cabeza Femoral

IMC: Índice de Masa Corporal de Quetelet

Hb Preop: Hemoglobina Preoperatoria

\#: Se ha aplicado la prueba t de Student

En la evaluación del sangrado perioperatorio (Tabla 2) no hemos detectado diferencias significativas entre ambos grupos en cuanto a los valores de hemoglobina a las 6 ni a las 48 horas, ni en los descensos de los valores de hemoglobina desde el preoperatorio hasta las 6 y 48 horas. Tam- poco el número de pacientes transfundidos ni la tasa de concentrados de hematíes transfundidos por paciente mostraron diferencias significativas entre ambos tipos de abordaje. Por último, el drenaje quirúrgico resultó similar en ambos grupos, sin diferencias significativas.

Tabla 2. Análisis del sangrado perioperatorio y velocidad de recuperación

\begin{tabular}{|c|c|c|c|c|c|c|c|c|c|}
\hline & $\begin{array}{c}\mathrm{Hb} 6 \mathrm{~h} \\
\text { (g/dl) }\end{array}$ & $\begin{array}{c}\text { Hb } \\
\text { Preop-6h } \\
\text { (g/dl) }\end{array}$ & $\begin{array}{c}\mathrm{Hb} 48 \mathrm{~h} \\
\text { (g/dl) }\end{array}$ & $\begin{array}{c}\text { Hb } \\
\text { Preop- } \\
48 h \\
\text { (g/dl) }\end{array}$ & \begin{tabular}{|c|} 
№ Pac \\
Transfundidos
\end{tabular} & $\begin{array}{c}\mathrm{CH} / \\
\text { Paciente }\end{array}$ & $\begin{array}{c}\text { Drenaje } \\
\text { (mI) }\end{array}$ & $\begin{array}{c}\text { Deambulación } \\
\text { (días) }\end{array}$ & $\begin{array}{c}\text { Estancia } \\
\text { (días) }\end{array}$ \\
\hline $\begin{array}{c}\text { Grupo } \\
\text { PMIS }\end{array}$ & $\begin{array}{l}10,98 \\
(1,28)\end{array}$ & $\begin{array}{c}3,43 \\
(0,94)\end{array}$ & $\begin{array}{l}10,32 \\
(1,27)\end{array}$ & $\begin{array}{c}4,14 \\
(1,48)\end{array}$ & 11 & $\begin{array}{c}0,80 \\
(1,08)\end{array}$ & $\begin{array}{c}250 \\
(199,74)\end{array}$ & $\begin{array}{c}2,22 \\
(1,41)\end{array}$ & $\begin{array}{c}4,87 \\
(1,03)\end{array}$ \\
\hline $\begin{array}{c}\text { Grupo } \\
\text { PEST }\end{array}$ & $\begin{array}{l}10,86 \\
(2,03)\end{array}$ & $\begin{array}{c}3,06 \\
(1,50)\end{array}$ & $\begin{array}{c}9,70 \\
(1,51)\end{array}$ & $\begin{array}{c}4,48 \\
(1,73)\end{array}$ & 16 & $\begin{array}{c}1,04 \\
(1,09)\end{array}$ & $\begin{array}{c}338,04 \\
(174,93)\end{array}$ & $\begin{array}{c}4,48 \\
(1,38)\end{array}$ & $\begin{array}{c}8,44 \\
(2,67)\end{array}$ \\
\hline Sig. (p) & $0,900^{\#}$ & $0,64^{\#}$ & $0,352^{\#}$ & $0,181^{\#}$ & 0,190 & 0,129 & $0,240^{\#}$ & $\begin{array}{c}<\mathbf{0 0 0 0 1}^{\#} \\
\text { IC95\% }^{\prime} \\
(1,3-2,2)\end{array}$ & $\begin{array}{c}<\mathbf{0 , 0 0 1}^{\#} \\
\text { IC95\% } \\
(1,5-5,6)\end{array}$ \\
\hline
\end{tabular}

Entre paréntesis la desviación estándar

LMIS: grupo de abordaje posterior mínimamente invasivo

LEST: grupo de abordaje posterior estándar

Hb: Hemoglobina; Hb Preop-6h: Descenso de hemoglobina desde el preoperatorio hasta las 6 horas del postoperatorio;

Hb Preop-48h: Descenso de Hemoglobina desde el preoperatorio hasta las 48 horas del postoperatorio

№Pac: Número de Pacientes

$\mathrm{CH} /$ Paciente: Concentrados de hematíes transfundidos por paciente.

\#: Se ha aplicado la prueba t de Student

n: Se ha aplicado la prueba U de Mann-Whitney

IC95\%: Intervalo de confianza del 95\% para la diferencia 
Para el estudio del dolor postoperatorio hemos evaluado, en primer lugar, el número de pacientes que precisaron analgésicos para tratar el dolor los días 1 y 2 del postoperatorio. En el día 1 del postoperatorio un total de 23 pacientes del grupo MIS y 22 del grupo estándar precisaron la administración de analgésicos para tratar el dolor $(\mathrm{p}=1)$, mientras que en el día 2 del postoperatorio fueron 13 pacientes del grupo MIS y 22 del grupo estándar cada grupo (p 0,012). Además, el número de pacientes que precisó tomar opioides para controlar el dolor fue significativamente menor en el grupo de abordaje mínimamente invasivo tanto en el día 1 ( 3 vs 8 ) como en el día 2 ( 1 vs 5) ( $\mathrm{p}=0,009$ y $\mathrm{p}=0,008$ respectivamente). Otro parámetro estudiado fue la cantidad de metamizol (nolotil®) administrado por paciente en los días 1 y 2; en el día 1 del postoperatorio se administraron $4.080 \mathrm{mg}$ metamizol/paciente (desviación estándar $621,2)$ en el grupo de pacientes intervenido mediante abordaje mínimamente invasivo mientras que en el grupo intervenido mediante abordaje estándar fueron $4.240 \mathrm{mg}$ metamizol/paciente (DE 856,34). Esta diferencia no fue estadísticamente significativa ( $p=0,045)$. En el día 2 del postoperatorio se administraron $2.320 \mathrm{mg}$ de metamizol/ paciente (DE 841,6) en el grupo PMIS mientras que el grupo PEST fueron $4.640 \mathrm{mg}$ metamizol/paciente (DE 364,1); esta diferencia fue estadísticamente significativa $(\mathrm{p}=0,002$, intervalo de confianza de 451,2-4.188,8).

$\mathrm{Al}$ igual que en el caso del dolor postoperatorio, el estudio del tiempo de recuperación también mostró diferencias significativas. Como puede observarse en la tabla 2 tanto el inicio de la deambulación (día en el que lo pacientes fueron capaces de caminar 10 pasos seguidos) como la estancia postoperatoria fueron significativamente mejores en el grupo de pacientes intervenidos mediante abordaje mínimamente invasivo $(p<0,001)$. Si interpretamos el límite inferior del intervalo de confianza del 95\% como la mínima diferencia esperada entre ambos grupos, el inicio de la deambulación se produjo, al menos, 1,31 días antes en el grupo de pacientes intervenido mediante abordaje mínimamente invasivo mientras que la estancia postoperatoria fue, al menos, 1,03 días menor en el grupo de pacientes intervenido mediante abordaje MIS.

El tiempo quirúrgico medido de incisión cutánea a cierre de la piel fue 120,1 minutos en el grupo PMIS (DE 29,83) y 115,6 minutos (DE 24,46) en el grupo de pacientes intervenidos mediante abordaje convencional. Esta diferencia no fue estadísticamente significativa $(\mathrm{p}=0,123)$.

En la tabla 3 se exponen los resultados de las variables seleccionadas para evaluar la orientación y el ajuste de los componentes. Como puede observarse en dicha tabla 3 no se detectaron diferencias significativas en el ángulo del componente acetabular respecto a la línea bi-isquiática, ni en el porcentaje de cótilos colocados horizontales $\left(<35^{\circ}\right)$, neutros $\left(35-45^{\circ}\right)$ o verticales $\left(>45^{\circ}\right)$. Tampoco se detectaron diferencias en cuanto al ángulo del componente femoral respecto al eje diafisario ni en cuanto al porcentaje de vástagos colocados en varo $\left(<177^{\circ}\right)$, neutros $\left(177-183^{\circ}\right)$ o en valgo $\left(>183^{\circ}\right)$. El relleno metafisario y el relleno diafisario no mostraron diferencias entre los dos grupos. Por último, en cuanto a la dismetría clínica en el postoperatorio, ésta fue de 1,7 mm (DE 9,92) en el grupo de pacientes intervenidos mediante abordaje mínimamente invasivo y de $3,7 \mathrm{~mm}$ (DE 8,90) en el grupo de abordaje convencional; esta diferencias no resultó estadísticamente significativa ( $\mathrm{p}=0,064$, $\mathrm{t}$ de Student). 
Tabla 3. Resultado de orientación y ajuste de los componentes y del Harris Hip Score a los 3 y 12 meses

\begin{tabular}{|c|c|c|c|c|c|c|c|c|}
\hline & $\begin{array}{c}\text { o'Cotilo } \\
\text { (grados) }\end{array}$ & $\begin{array}{c}\text { Inclinación } \\
\text { Cotilo }\end{array}$ & $\begin{array}{l}\text { oVástago } \\
\text { (grados) }\end{array}$ & $\begin{array}{c}\text { Inclinación } \\
\text { Vástago }\end{array}$ & $\begin{array}{c}\text { Relleno } \\
\text { Metafisario }\end{array}$ & $\begin{array}{c}\text { Relleno } \\
\text { diafisario }\end{array}$ & $\begin{array}{l}\text { HHS } 3 \\
\text { meses }\end{array}$ & $\begin{array}{c}\text { HHS } 12 \\
\text { meses }\end{array}$ \\
\hline $\begin{array}{c}\text { Grupo } \\
\text { PMIS }\end{array}$ & $\begin{array}{l}42,10^{\circ} \\
(6,82)\end{array}$ & $\begin{array}{c}3 \mathrm{H} \\
13 \mathrm{~N} \\
8 \mathrm{~V}\end{array}$ & $\begin{array}{l}179,9^{\circ} \\
(1,39)\end{array}$ & $\begin{array}{l}1 \mathrm{VR} \\
23 \mathrm{~N} \\
1 \mathrm{VL}\end{array}$ & $\begin{array}{c}0,81 \\
(0,14)\end{array}$ & $\begin{array}{c}0,91 \\
(0,07)\end{array}$ & $\begin{array}{l}96,28 \\
(4,36)\end{array}$ & $\begin{array}{c}94,2 \\
(4,13)\end{array}$ \\
\hline $\begin{array}{c}\text { Grupo } \\
\text { PEST }\end{array}$ & $\begin{array}{l}44,68^{\circ} \\
(4,79)\end{array}$ & $\begin{array}{c}0 \mathrm{H} \\
15 \mathrm{~N} \\
10 \mathrm{~V}\end{array}$ & $\begin{array}{l}179,8^{\circ} \\
(1,51)\end{array}$ & $\begin{array}{l}2 \mathrm{VR} \\
22 \mathrm{~N} \\
1 \mathrm{VL}\end{array}$ & $\begin{array}{c}0,85 \\
(0,11)\end{array}$ & $\begin{array}{c}0,90 \\
(0,07)\end{array}$ & $\begin{array}{l}94,28 \\
(5,12)\end{array}$ & $\begin{array}{c}95,6 \\
(3,69)\end{array}$ \\
\hline Sig. (p) & $0,317^{\#}$ & 0,121 & $0,097^{\mathrm{n}}$ & 0,323 & $0,760^{\#}$ & $0,056^{\mathrm{n}}$ & $0,315^{\#}$ & $0,091^{\mathrm{n}}$ \\
\hline
\end{tabular}

Entre paréntesis la desviación estándar

LMIS: grupo de abordaje posterior mínimamente invasivo

LEST: grupo de abordaje posterior estándar

${ }^{\circ}$ Cotilo: Ángulo de Inclinación del componente acetabular respecto a la línea bi-isquiática

@Vástago: Ángulo de Inclinación del componente femoral respecto al eje diafisario femoral HHS: Harris Hip Score

\#: Se ha aplicado la prueba t de Student

n: Se ha aplicado la prueba U de Mann-Whitney

En el capítulo de complicaciones, en el grupo de pacientes intervenidos mediante abordaje posterior MIS se produjeron las siguientes complicaciones: un caso de seroma de la herida que no precisó tratamiento y un caso de luxación tras una caída por una crisis epiléptica a las 24 horas de la intervención que se solucionó de forma conservadora sin recidivas. Por otra parte, en el grupo de pacientes intervenidos mediante abordaje convencional no se detectó ninguna complicación. Tras analizar estadísticamente estos datos no se halló significación estadística en esta diferencia ( $p>0,05$, Chi-cuadrado).

En la evaluación del resultado funcional hemos utilizado la escala de cadera de Harris medida a los tres y 12 meses de la intervención quirúrgica. En ambas mediciones los resultados de los dos grupos fueron excelentes ( $>90$ puntos) sin que se detectaran diferencias significativas entre ambos grupos. Los valores de la escala de Harris de ambos grupos a los tres y 12 meses se exponen en la tabla 3.

Por último, nos propusimos estudiar el impacto económico de nuestro estudio en nuestro hospital. Teniendo en cuenta que se había conseguido una disminución de la estancia hospitalaria de 3,57 días de media con la cirugía mínimamente invasiva en comparación con la técnica convencional y que el coste de cada día de ingreso era de 135,6 euros, se ha conseguido un ahorro de 417,1 euros por paciente. El coste por proceso de artroplastia total de cadera era de $9.217,3$ euros; por lo tanto, se ha conseguido reducir el coste por proceso en un $5,25 \%$. En total, al haber aplicado el proceso de cirugía MIS a 25 pacientes, el ahorro total fue de 12.105,75 euros.

\section{DISCUSIÓN}

La cirugía mínimamente invasiva en artroplastia total de cadera está de plena actualidad. Sin embargo, apenas existen trabajos con un diseño metodológico que pueda proporcionar un alto nivel de evidencia científica ${ }^{9}$. Para intentar introducir algo más de claridad en este tema hemos diseñado un estudio prospectivo y aleatorizado en el que ambos grupos resultaron homogéneos en todas las variables preoperatorias estudiadas; por ello creemos que se pueden extraer conclusiones válidas y potentes en nuestro medio de nuestros resultados.

A la hora de comparar nuestros resultados con los de la literatura existente, 
hemos decidido clasificar los estudios en función de su nivel de evidencia utilizando para ello las recomendaciones de la $U S$ Agency for Health Care Policy Research ${ }^{12}$, que clasifica los diferentes trabajos en los siguientes niveles de evidencia:

- Ia: metaanálisis de estudios prospectivos aleatorizados.

- Ib: estudio prospectivo aleatorizado.

- Ila: estudio prospectivo sin aleatorizar.

- IIb: estudio retrospectivo o de cohortes.

- III: estudio descriptivo.

- IV: opinión de expertos. Según esta clasificación, nuestro estudio gozaría de un nivel de evidencia Ib.

En nuestro estudio -al igual que otros autores $^{13-16}$ en trabajos publicados con nivel de evidencia Ib-, no hemos encontrado diferencias entre los abordajes posterior MIS y convencional al evaluar el sangrado perioperatorio. Ninguno de ellos encontró un menor sangrado perioperatorio en el grupo de pacientes intervenidos mediante cirugía mínimamente invasiva. Además, nuestros resultados también coinciden con los de Woolson y col ${ }^{17}$ (IIb) los cuales tampoco encontraron diferencias significativas. Sin embargo, Panisello y col ${ }^{18}$ (nivel de evidencia Ib) detectaron un menor sangrado en el grupo de abordaje mínimamente invasivo; sin embargo, en sus resultados se adivinan algunas contradicciones. Así detectaron una mayor caída de los niveles de hemoglobina y hematocrito a las 6 horas de la intervención (que no se concretaron a las 24 y 48 horas) y, sin embargo, el drenaje quirúrgico en el mismo periodo de tiempo fue sólo $20 \mathrm{ml}$ mayor en el grupo de abordaje convencional. Además, no especificaron si los niveles de hemoglobina y hematocrito mostraron diferencias significativas a las 6 horas. Exceptuando estos trabajos, los demás que demostraron mayor sangrado en el grupo de pacientes intervenidos mediante abordaje convencional en comparación con el abordaje mínimamente invasivo son de menor nivel de evidencia. Así Chung y col' ${ }^{19}$, Nakamura y col $^{20}$ y Scul- co y col$^{21}$, todos de nivel de evidencia IIa, presentaron un menor sangrado perioperatorio con la cirugía mínimamente invasiva. Por último, Khan y coll ${ }^{22}$, Goldstein y $\mathrm{col}^{23}$, Wenz y col $^{24}$ y Wright y $\mathrm{col}^{25}$, trabajos de nivel de evidencia IIb, detectaron un menor sangrado con la utilización de las técnicas de cirugía mínimamente invasiva. Khan y $\mathrm{col}^{22}$ y Goldstein y $\mathrm{col}^{23}$ (ambos IIb) detectaron una menor pérdida sanguínea estimada, pero este hallazgo no se tradujo en una mayor caída de la hemoglobina ni en una mayor tasa de transfusiones. Wright y $\mathrm{col}^{25}$ basaron sus conclusiones únicamente en la pérdida sanguínea estimada (sólo $20 \mathrm{ml}$ de diferencia real); por último Wenz y col$^{24}$ demostraron una menor pérdida sanguínea estimada y una menor tasa de transfusiones con la vía posterior mínimamente invasiva; sin embargo, este estudio presenta los problemas metodológicos de comparar la vía posterior MIS con la vía lateral convencional y, además, el IMC resultó significativamente menor en el grupo de abordaje lateral convencional.

En el estudio del dolor postoperatorio hemos detectado un menor consumo analgésico en el grupo de abordaje mínimamente invasivo. En este punto nuestros resultados coinciden con los de Dorr y col $^{13}$ (Ib) que evaluaron el dolor postoperatorio mediante la escala analógico-visual del dolor (EVA). Sin embargo, con una evaluación también muy exhaustiva, Ogonda y $\mathrm{col}^{16}$ (Ib) no consiguieron demostrar un menor dolor postoperatorio con la cirugía mínimamente invasiva. Ya en otro nivel de evidencia Chung y colli (IIa) no encontraron diferencias en el tiempo que los pacientes mantenían la bomba de analgesia.

El abordaje posterior MIS presentó en nuestro estudio una mayor velocidad de recuperación, demostrada en un inicio más precoz de la deambulación así como en una menor estancia hospitalaria. Nuevamente, nuestros resultados coinciden con los de Dorr y col $^{13}$ (Ib), Chimento y Sculco ${ }^{21,15}$ (IIa, Ib), Chung y col ${ }^{19}$ (IIa), Khan y col$^{22}$ y Wenz y col $^{24}$ (IIb), ya que todos ellos detectaron una mayor velocidad de recuperación con la cirugía mínimamente invasiva. Sin em- 
bargo, los trabajos de Ogonda y col $^{16}$ (Ib), Lawlor y col $^{26}$ (Ib), Woolson y col $^{17}$ (IIb), Wright y col${ }^{25}$ (IIb) y Murphy y col${ }^{4}$ no encontraron diferencias significativas.

No hemos detectado diferencias significativas en lo relativo al tiempo quirúrgico entre el abordaje posterior mínimamente invasivo y el abordaje posterior convencional. Nuestros resultados coinciden con Dorr y coll3 y Ogonda y col ${ }^{16}$ (ambos Ib), con los estudios prospectivos no aleatorizados de nivel IIa de Chung y col $^{19}$ y Murphy y $\operatorname{col}^{4}$ y con los estudios de nivel de evidencia IIb de Wright y col $^{25}$ y Kahn y coll $^{22}$. Sin embargo, los estudios de Kim y col $^{14} \mathrm{y}$ Sculco y col $^{15}$, ambos de nivel de evidencia Ib y Wenz y col ${ }^{24}$ (IIb), detectaron diferencias entre ambos abordajes; sin embargo, éstas fueron a favor de la cirugía mínimamente invasiva.

Otro de los posibles inconvenientes de la cirugía mínimamente invasiva es la peor orientación de los componentes. No hemos detectado ninguna diferencia en la orientación del componente acetabular y femoral entre el abordaje mínimamente invasivo posterior y el convencional. No hemos encontrado en la literatura ningún trabajo que haya detectado peor orientación de los componentes con el abordaje posterior MIS. Tampoco hemos encontrado diferencias significativas en cuanto al ajuste de los componentes, tampoco ninguno de los trabajos consultados detectó un peor ajuste de los componentes.

Como se ha expuesto en el capítulo de resultados no hemos encontrado diferencias significativas en la tasa de ninguna de las complicaciones encontradas entre los abordajes lateral MIS y convencional. Ningún estudio de los consultados detectó una mayor tasa de complicaciones con la cirugía MIS e incluso el estudio de Murphy y $\mathrm{col}^{4}$ detectó menor tasa de complicaciones con la cirugía MIS.

En nuestro estudio no hemos encontrado diferencias significativas en el resultado funcional a los tres y doce meses de la intervención quirúrgica, habiendo valorado la puntuación del Harris Hip Score. Aunque existen autores que demuestran un mejor resultado funcional como Wright y $\mathrm{col}^{25}$ (IIb), Sculco y col' ${ }^{21}$ (IIb), Khan y col' ${ }^{22}$ (IIb) y Murphy y col$^{4}$ (IIb), estos trabajos son de metodología cuestionable. El hecho es que ningún trabajo de nivel de evidencia Ib, como son los trabajos de Kim y col ${ }^{14}$, Dorr y $\mathrm{col}^{13}$, Ogonda y $\mathrm{col}^{16}$, Panisello y $\mathrm{col}^{18} \mathrm{y}$ Sculco y col$^{21}$, ha sido capaz de demostrar un mejor resultado funcional ni a medio ni a largo plazo.

En resumen, el abordaje posterior mínimamente invasivo ha conseguido disminuir el dolor postoperatorio y acelerar la recuperación en comparación con el abordaje convencional sin comprometer el tiempo quirúrgico, la orientación o ajuste de los componentes ni aumentar la tasa de complicaciones, con un resultado funcional similar a las técnicas convencionales y un impacto económico favorable a la cirugía mínimamente invasiva.

\section{BIBLIOGRAFÍA}

1. Inaba Y, Dorr LD, Wan Z, Sirianni L, Boutary M. Operative and patient care techniques for posterior mini-incision total hip arthroplasty. Clin Orthop Rel Res 2005; 441: 104-114.

2. Berger RA, Jacobs JJ, Meneghini RM, Della Valle C, Paprosky W, Rosenberg AG. Rapid rehabilitation and recovery with minimally invasive total hip arthroplasty. Clin Orthop Rel Res 2004; 429: 239-223.

3. Howell JR, Garbuz DS, Duncan CP. Minimally invasive hip replacement: rationale, applied anatomy, and instrumentation. Orthop Clin N Am 2004; 35: 107-118.

4. Murphy SB, Ecker TM, TANnAst M. THA performed using conventional and navigated tissue-preserving techniques. Clin Orthop Rel Res 2006; 44: 160-167.

5. O`BriEn DAL, Rorabeck CH. The mini-incision lateral approach in primary total hip arthroplasty. Clin Orthop Rel Res 2005; 441: 99-103.

6. MALIK A, DoRR LD. The science of minimally invasive total hip arthroplasty. Clin Orthop Rel Res 2007; 463: 74-84.

7. Chimento GF, Pavone V, Sharrock N, Kahn B, CAHILL J, Sculco TP. Minimally invasive total hip arthroplasty-A prospective randomized study. J Arthroplasty 2005; 20: 139-144.

8. Goldstein WM, Branson JJ, Berland KA, Gordon AC. Minimal-incision total hip arthroplasty. J Bone Joint Surg Am 2003; 85 (Suppl 4): 33-38. 
9. Labovitch RS, Bozic KJ, Hansen E. An evaluation of information available on the internet regarding minimally invasive hip arthroplasty. J Arthroplasty 2006; 21: 1-5.

10. Sculco TP, Jordan LC, WALTER WL. Minimally invasive total hip arthroplasty: the Hospital for Special Surgery experience. Orthop Clin N Am 2004; 35: 16-20.

11. Barrack RL, Milroy RD JR, Harris WH. Improved cementing techniques and femoral loosening in young patients with hip arthroplasty. A 12-years radiographic review. J Bone Joint Surg Br 1992; 74: 385-389.

12. Saha S, Hoerger TJ, Pignone MP, Teustsch SM, Helfand M, Mandelblatt JS, for the Cost Work Group of the Third U.S. Preventive Services Task Force. The art and science of incorporating cost effectiveness into evidence-based recommendations for clinical preventive services. Am J Prev Med 2001; 20 (Suppl 3): $36-43$.

13. Dorr LD, Maheshwari AV, Long WT, Wan Z, SiRIANNI LE. Early pain relief and fuction after posterior minimally invasive and conventional total hip arthroplasty. A prospective, randomized, blinded study. J Bone Joint Surg Am 2007; 89: 1153-1160.

14. КІм YH. Comparison of primary total hip arthroplasties performed with a minimally invasive technique or a standard technique-A prospective and randomized study. J Arthroplasty 2006; 21: 1092-1098.

15. Bottner FB, Delgado S, Sculco TP. Minimally invasive total hip replacement: the posterolateral approach. Am J Orthop 2006; 35: 218224.

16. Ogonda L, Wilson R, Archbold P, Lawlor M, HumPHREYS P, O`BRIEN S et al. A minimal-incision technique in total hip arthroplasty does not improve early postoperative outcomes-A prospective, randomized controlled trial. J Bone Joint Surg 2005; 87: 701-714.

17. Woolson ST, Mow CS, Syquia JF, Lannin JV, SCHURMAN DJ. Compasiron of primary total hip replacement performed with a standard incision or a mini-incision. J Bone Joint Surg Am 2004; 86: 1353-1358.

18. Panisello-Sebastiá JJ, Canales-Cortés V, HerreroBarcos L, Herrera-Rodríguez A, Mateo-Agudo J, Marínez-Martín AA. Efectividad de la cirugía mínimamente invasiva de incisión única postero-lateral en artroplastia total de cadera. Rev Ortop Traumatol 2006; 21: 425-430.

19. Chung WK, LiU D, Foo LSS. Mini-incision total hip replacement-Surgical technique and early results. J Orthop Surg 2004; 12 : 19-24.

20. Nakamura S, Matsuda K, Arai N, Wakimoto N, MATSUSHITA T. Mini-incision posterior approach for total hip arthroplasty. Int Orthop 2004; 28: 214-217.

21. Chimento GF, Pavone V, Sharrock N, Kahn B, CaHILL J, Sculco TP. Minimally invasive total hip arthroplasty-A prospective randomized study. J Arthroplasty 2005; 20: 139-144.

22. Khan RJK, Fick D, Khoo P, Yao F, Nivbrant B, Wood D. Less invasive total hip arthroplastyDescription of a new technique. J Arthroplasty 2006; 21: 1038-1046.

23. Goldstein WM, Branson JJ, Berland KA, Gordon AC. Minimal-incision total hip arthroplasty. J Bone Joint Surg Am 2003; 85 (Suppl 4): 33-38.

24. Wenz JF, GuRKan I, Jibodh SR. Mini-incision total hip arthroplasty: a comparative assessment of perioperative outcomes. Orthopedics 2002; 25: 1031-1043.

25. Wright JM, Crockett HC, Delgado S, Lyman S, Madsen M, Sculco TP. Mini-incision for total hip arthroplasty-A prospective, controlled investigation with 5-year follow-up evaluation. J Arthroplasty 2004; 19: 538-544.

26. LaWlor M, Humphreys P, Morrow E, Ogonda L, Bennett D, Elliott D. Comparison of early postoperative functional levels following total hip replacement using minimally invasive versus standard incisions. A prospective randomized blinded study. Clin Rehabil 2005; 19: 465-474. 
